\title{
Domain Structure of Co/Pd Multilayers
}

\author{
Jarmila Šimšvá and Richard Gemperle \\ Institute of Physics, Academy of Science, Cukrovarnická 10, Praha 6, Czech Republic \\ Vladimir Kamberský, Steffen Porthun, Poul de Haan and Cock Lodder \\ MESA Research Institute, University of Twente, P.O.Box 217, 7500 AE Enschede, The Netherlands
}

\begin{abstract}
The observations of submicron domain structure of Co/Pd multilayers at various parts of the M-II loop and after different magnetization cycles designed to approach the globalequilibrium domain width are reported. The wall energy densities were estimated from comparison of the measured equilibrium domain width with the anhysteretic model predictions and also compared with the wall energy densities determined from the slope of major M-H loop.
\end{abstract}

\section{INTRODUCTION}

Co-based multilayers (MLs), consisting of periodically alternating ferromagnetic $\mathrm{Co}$ and non magnetic $\mathrm{Pd}$ or $\mathrm{Pt}$ layers represent a very promising material for high density magnetooptical recording, and an extensively studied class of low dimensional systems: Co layers are typically 0.2 to $0.8 \mathrm{~nm}$ thick.

In spite of the large progress in the MLs, only few papers are devoted to observation of their domain structure. Hashimoto [1,2] observed remanent stripe domain structure in the $\mathrm{Co} / \mathrm{Pd}$ and $\mathrm{Co} / \mathrm{Pt}$ MLs using Kerr microscopy. Honda $[3,4]$ used Bitter technique (with an optical microscope) to observe stripe domain structure of $\mathrm{Co} / \mathrm{Pt} \mathrm{ML}$ on the descending branch of major hysteresis loop, and from this non equilibrium period calculated the wall energy density $\left(6-8 \mathrm{~mJ} / \mathrm{m}^{2}\right)$. For very thin Co/Pt MLs Lorentz electron microscopy [6] was used to visualize stripe domain structure. Donnet [7] observed remanent domain structure of $\mathrm{Co} / \mathrm{Pt} \mathrm{MLs}$ (after different field application) using differential phase contrast mode of Lorentz microscopy. The observed remanent domain widths were compared with the predictions of the model $[8,4]$ balancing the magnetostatic energy; such comparison allows to estimate the specific wall energy.

Comparison of the observed hysteresis loops with the model magnetization curves $[8-10,4]$ is problematic due

Manuscript received May 31, 1993. to the relative smallness of magnetostatic fields compared to the coercivity [5].

In a previous paper [10] we discussed the universal scaling of the model predictions and the dominant role of inter-layer magnetostatic interactions. The model predictions were compared with the domain observations on two Co/Pd samples.

In this work we present observations of the submicron domain structure on three $\mathrm{Co} / \mathrm{Pd}$ multilayers at various parts of the $\mathrm{M}-\mathrm{H}$ loop and after various magnetization cycles designed to approach the global-equilibrium domain size comparable to the anhysteretic model predictions. We discuss the wall energy and exchange constant estimated in this way and also compare the model predictions with the observed slopes of the hysteresis loops.

\section{EXPERIMENTS}

\section{A. Samples and magnetic measurements}

Samples of Co/Pd MLs were prepared by rf sputtering in Ar, M-H loops were measured by VSM (M, is magnetization of $\mathrm{Co}$ ) and anisotropy constants $\mathbf{K}_{\text {eff }}$ by a torque magnetometer [11]. In Table I the parameters of three Co/Pd MLs with the same thickness of $t_{p d}=1.4$ $\mathrm{nm}$ and with different thickness of Co layer, $t_{c o}$ (number of bilayers $\mathrm{N}=25$; total thickness, $\mathrm{T}$ ) and different coercivity, $\mathrm{H}_{c}$ are summarized. For comparison with the stripe-model predictions we also list in Table III the slopes of $\mathrm{dM} / \mathrm{dH}$ of the measured $\mathrm{M}-\mathrm{H}$ loops [11]: (i) on the descending branch near $\mathrm{H}_{c}$ (desc) and (ii) on the nearly linear parts of the ascending branch near 50\% magnetization $M_{\text {, (asc). }}$

\section{B. Domain observations - CSEM method}

We measured the domain widths using the CSEM method (observation of dried Bitter patterns in scanning electron microscope) [12]. The measurements of domain 
TABLE I

THICKNESS AND MAGNETIC PARAMETERs of Co/Pd MLs

\begin{tabular}{lccccc}
\hline Sample No. & $\mathrm{t}_{c o}$ & $\mathrm{~T}$ & $\mathrm{M}_{\mathbf{c}}$ & $\mathrm{H}_{\mathrm{c}}$ & $\mathrm{K}_{\mathrm{eff}}$ \\
& $(\mathrm{nın})$ & $(\mathrm{nm})$ & $(\mathrm{kA} / \mathrm{m})$ & $(\mathrm{kA} / \mathrm{m})$ & $\left(\mathrm{kJ} / \mathrm{m}^{3}\right)$
\end{tabular}

$\begin{array}{rrrrrrr}1 & 910814-1 & 0.85 & 56.3 & 1850 & 36 & 42 \\ 2 & 910807-1 & 0.45 & 46.5 & 2026 & 96 & 1006 \\ 3 & 910808-1 & 0.23 & 41.0 & 2445 & 152 & 1615\end{array}$
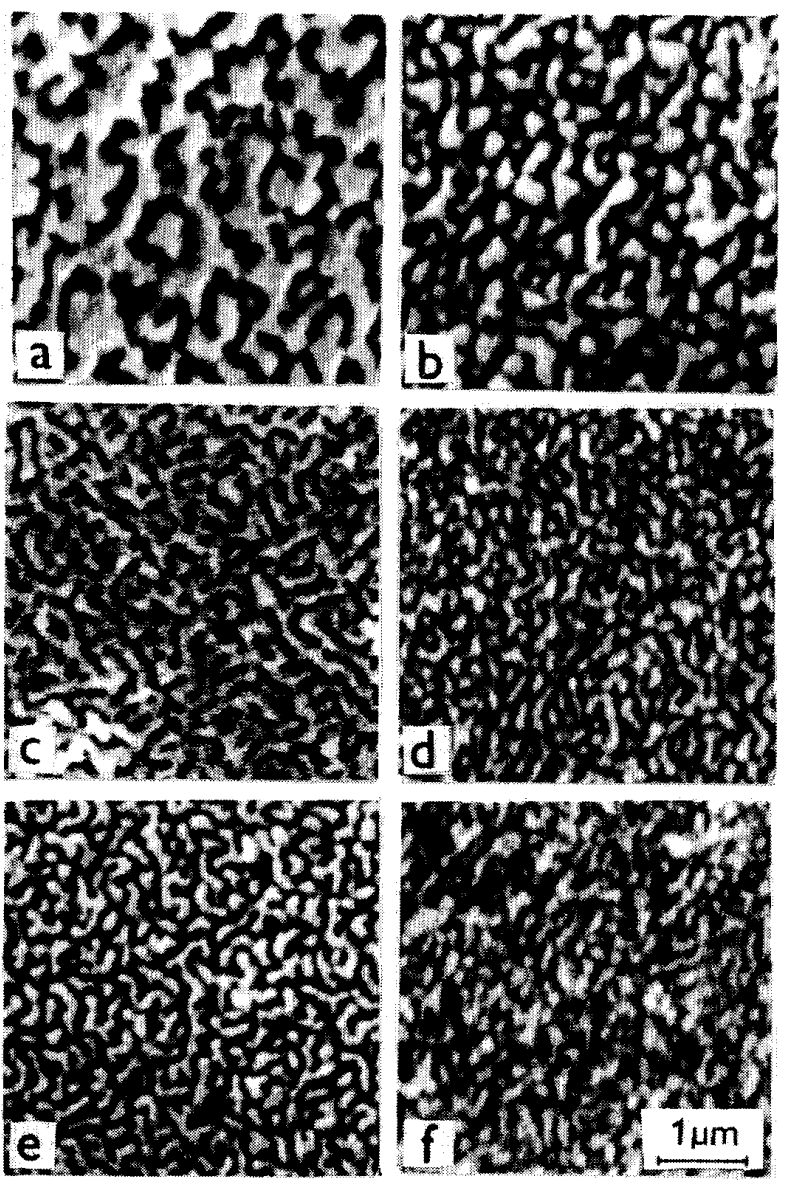

Fig. 1 The domain structures of sample No. 1: a-descending branch $160 \mathrm{kA} / \mathrm{m}$, b-descending $80 \mathrm{kA} / \mathrm{m}$, c-ascending $240 \mathrm{kA} / \mathrm{m}, d$-ac demagnetized, e-minor loop $H_{m}= \pm 160 \mathrm{kA} / \mathrm{m}, \mathrm{f}$-de demagnetized. period $\mathrm{p}$ were performed at various points of the major M-H loop. In addition, we used several ways of demagnetization in order to estimate the domain width, $D_{0}=p / 2$ in states approaching global equilibrium. For "medium" coercivity sample No. 1 the same domain periods were obtained by ac demagnezation, by cycling on minor loops [13] and also by dc demagnetization (Fig. 1 d,e,f and Table II). On the two high coercivity samples No. 2 and 3 we obtained the same domain periods (close to global equilibrium state) by dc demagnetization and by cycling on minor loops with maximum field $\mathrm{H}_{\mathrm{m}}=160 \mathrm{kA} / \mathrm{m}$ (Fig. 2e,f and Table II). However, a lower amplitude $\mathrm{H}_{\mathrm{m}}=120 \mathrm{kA} / \mathrm{m}$ was not high enough to reach the equilibrium domain width in these high coercivity samples (Fig. 2d and Table II).
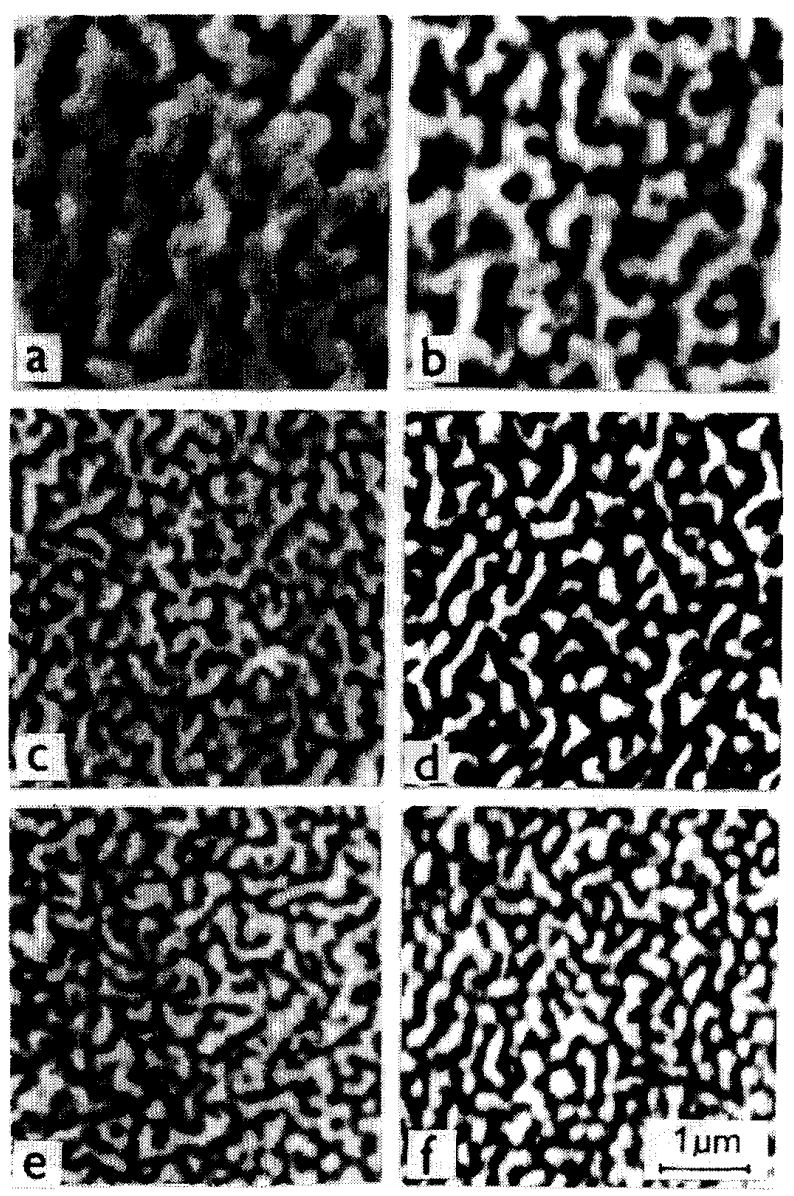

Fig. 2 The domain structures of sample No. 2: a-descending branch $-92 \mathrm{kA} / \mathrm{m}$, b-descending $-96 \mathrm{kA} / \mathrm{m}$, c-ascending $-160 \mathrm{kA} / \mathrm{m}$, d-minor loop $H_{m}= \pm 120 \mathrm{kA} / \mathrm{m}$, e-minor loop $H_{m}= \pm 160 \mathrm{kA} / \mathrm{m}$, f-dc demagnetized. 


\section{TABLE II}

DOMAIM PERIODS ON THE MAJOR LOOP AND AFTER DEMAGNETIZATION

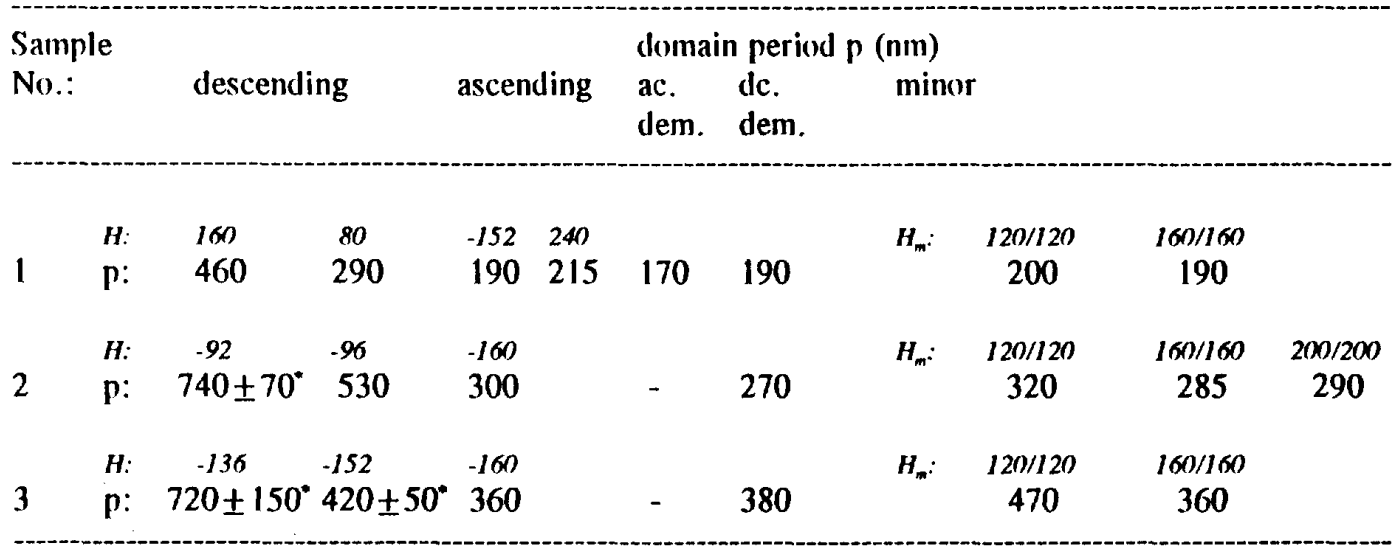

Domain periods, $p$ measured on major loop at different values of $\mathrm{H}(\mathrm{kA} / \mathrm{m})$ and after various demagnetization: ac dem.: slowly decreasing ac field of $\max 96 \mathrm{kA} / \mathrm{m}$ to zero, dried at 40 and $80 \mathrm{kA} / \mathrm{m}$ respectively.

de dem.: succesive decreasing de lield starting from $\pm 440 \mathrm{kA} / \mathrm{m}$ with step of $40 \mathrm{kA} / \mathrm{m}$ down to $+40 \mathrm{kA} / \mathrm{m}$ $160 / 160=$ minor loop at $H_{m}=160 \mathrm{kA} / \mathrm{m}$, (cycling 3 times) dried at $+160 \mathrm{kA} / \mathrm{m}$ and $/ \mathrm{or} 40 \mathrm{kA} / \mathrm{m}$.

The errors in the deternination of the domain period (mostly caused by the irregularity of the structure) were less than 20 nin with exception of cases marked by .

\section{Domain observations - Magnetic Force Microscopy}

Preliminary results obtained by Magnetic Force Microscopy, (Fig. 3) on sample No.1 demagnetized in decreasing rotating field show good agreement in the average domain width, $D_{o}$. The general appearance of the domain patterns is of quite regular stripes, which is ascribed to the way of demagnetazition in a decreasing field rotating in a plane perpendicular to the sample surface.

Fig. 3

MFM image of field grndient of the domain structure of sample No. 1 $\left(D_{n}=p_{0} / 2=\right.$ $=70 \pm 10 \mathrm{~nm}$ ).

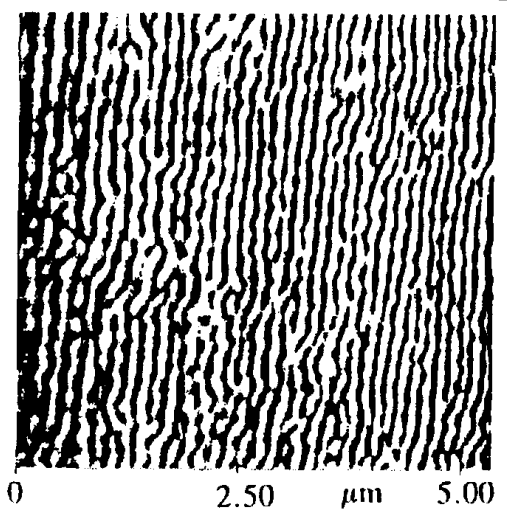

\section{DisCussion AND CONCLUSIONS}

The measured average values of domain width $D_{n}=p_{o} / 2$ presumably close to anhysteretic values are summarized in Table III. The predictions of the periodic stripe model are shown in Fig. $4[10]$; the experimental points are denoted by sample numbers $1,2,3$. (while the points 1', 2', 3' correspond to the same film parameters but for the number of bilayers $N$, reduced to 12 . Table III shows the wall energy densities $\sigma_{D}$ estimated

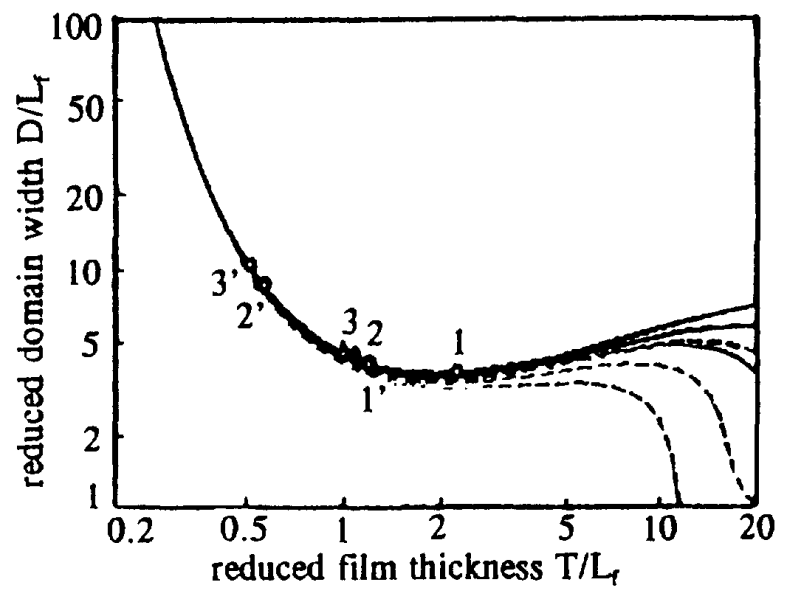

Fig. 4 Dependence of the domain width $D$ on film thickness $T$ [10\}; the nonnalizing factor $L_{t}=\left(\mathrm{N} \mathrm{t}_{\mathrm{co}_{0}} / \mathrm{T} .0 / 0.5 \mu_{0} \mathrm{M}_{\mathrm{i}}{ }^{2}\right)$ is the characteriatic length of the ML. Points 1, 2, 3 and the solid curves represent the experimental points and film paraneters $N=25$, the filling factors $\left(f=t_{C_{0}} / t_{C_{0}}+t_{p d}\right): f=0.37$ (lop curve), 0.24 and 0.14 ; the dashed curves and points $1^{\prime}, 2,3$ ' represent model predictions for the same film parameters but for number of bilayers $\mathrm{N}$, reduced to 12 . 
TABLE III

THE VALUES OF DOMAIN WALL ENERGY DENSITIES OF Co/Pd MLs ESTIMATED FROM THE DOMAIN WIDTH $\left(\sigma_{D}\right)$ AND FROM $\mathrm{dM} / \mathrm{dH}\left(\sigma_{\mathrm{desc}}\right)$

\begin{tabular}{lcccccccc}
$\begin{array}{l}\text { Sample } \\
\text { No.: }\end{array}$ & $\begin{array}{l}\mathrm{D}_{0} \\
(\mathrm{~nm})\end{array}$ & $\begin{array}{c}\mathrm{L}_{\mathrm{f}} \\
(\mathrm{nm})\end{array}$ & $\begin{array}{c}\sigma_{\mathrm{D}} \\
\left(\mathrm{mJ} / \mathrm{m}^{2}\right)\end{array}$ & $(\mathrm{dM} / \mathrm{dH})_{\mathrm{D}}$ & $(\mathrm{dM} / \mathrm{dH})_{\text {desc }}$ & $(\mathrm{dM} / \mathrm{dH})_{\text {asc }}$ & $\begin{array}{c}\sigma_{\text {dece }} \\
\left(\mathrm{mJ} / \mathrm{m}^{2}\right)\end{array}$ & $\begin{array}{c}\sigma_{\mathrm{K}} \\
\left(\mathrm{mJ} / \mathrm{m}^{2}\right)\end{array}$ \\
\hline 1 & $90 \pm 10$ & 24.6 & 19.6 & 6.4 & 5.8 & 4.4 & 17 & 20 \\
2 & $135 \pm 10$ & 35.8 & 22.1 & 17 & 25 & 10 & 25 & 24 \\
3 & $185 \pm 10$ & 42.7 & 22.5 & 39 & 77 & 26 & 33 & 25 \\
\hline
\end{tabular}

The equilibrium domain width $D_{0}$; the characteristic length, $L_{i}$.

\section{REFERENCES}

comparing the measured $\mathrm{D}_{\mathrm{o}}$ with the model.

Comparison of the slopes of the measured major M-H loops with the anhysteretic model predictions is more problematic [9]. Table III shows the slopes of the initial linear parts of $M(H),(d M / d H)_{D}$ obtained from the model using our values $\sigma=\sigma_{\mathrm{D}}$, and also the values of wall energy, $\sigma_{\text {dece }}$ needed to fit the experimental $(\mathrm{dM} / \mathrm{dH})_{\text {deac }}$. The agreement is reasonable in the relatively "soft" (medium coercivity) sample No. 1 but in the two "hard" (high coercivity) samples Nos. 2 and 3 the model $(\mathrm{dM} / \mathrm{dH}) \mathrm{D}$ lies between the very high $(\mathrm{dM} / \mathrm{dH})_{\text {deac }}$ (probably far from equilibrium, too close to high negative nucleation field) and the moderate $(\mathrm{dM} / \mathrm{dH})_{\text {asc }}$ (where magnetostatic effects are likely to prevail).

We also show in Table III the values $\sigma_{\mathrm{K}}=4\left(\mathrm{AK}_{\mathrm{u}}\right)^{1 / 2}$ obtained using assumed exchange constant $\mathrm{A}=10^{-11} \mathrm{~J} / \mathrm{m}$ and the measured $K_{v}=K_{\text {eff }}+0.5 \mu_{0} M_{a}^{2}$ [11]. "Micromagnetic" modelling analogous to [14] shows that the classical formula for $\sigma_{k}$ overestimates the wall energy in very thin layers with $K_{u}$ dominated by interface effects [10]; the agreement of $\sigma_{K}$ with the experimental $\sigma_{D}$ thus indicates that the effective exchange constant needed to fit the experimental results will be larger than that assumed above (corresponding to bulk cobalt).

The presented domain width measurements employing a variety of demagnetizatiom methods and colloid-SEM as well as MFM observations are in a good agreement with the predictions of the model of stripe domains penetrating the ML coherently, with domain wall energy density corresponding to bulk exchange and directly measured uniaxial anisotropy. The model predicts increasing equilibrium domain width with number of bilayers decreasing below the present $\mathrm{N}=\mathbf{2 5}$.
[1] S. Hnshimoto, and $\mathrm{Y}$. Ochiai, "Co/Pt and $\mathrm{Co} / \mathrm{Pd}$ multilayers as magneto-optical recording materialn," J. Mag. Mag. Mat., vol. 88, pp. 211-226, 1990.

[2] S. Hashimoto, Y Ochiai, and A. Aso, "Film thickness dependence of inngneto-optical and inagnetic properties in Co/Pt and Co/Pd multilayers," J. Appl. Phys., vol. 67, pp. 4429.4431, 1990.

[3] S. Honda, H. Tanimoto, and T. Kusuda, "Magnetizntion process and coencivity of sputtered Co/Pt multilayered films," IEEE Trans. Mag., vol. 26, pp. 2730-2732, 1990.

[4] S. Honda, Y. Ikegawa, and T. Kusuda, "Mngnetostatic energy and magnetization process in multilayers with perpendicular anisotropy," J. Mag. Mag. Mar., vol. 111, pp. 273-292, 1992.

[5] W.B. Zeper, H.W. van Kesteren, B.A.J. Jacobs, and J.H.M. Spruit, "Hysteresis, microstructure, and magneto-optical recording in Co/Pt and Co/Pd multilayers," J. Appl. Phys., vol. 70, pp. 2264-2271, August 1991.

[6] E.T.M. Lacey, and P.J.Grudy, "Magnetic and structural pmperties of nputter deponited Co/Pt multilayers," IEEE Trans. Mag., vol. 26, pp. 2356-2358, 1990.

[7] D.M. Donnet, J.N. Chapınan, H.W. van Kestem, and W.B. Zeper, "Investigation of domain structures in $\mathrm{Co} / \mathrm{Pt}$ multilayers by modified differential phase contrast microscopy," J. Mag. Mag. Mat. vol. 115, pp. 342.352, 1992.

[81 H.J.G. Draaisma, and W.J.M. de Jonge, "Magnetisation curves of Pd/Co multilayers with perpendicular anisotropy," J. Appl. Phys., vol. 62, pp. 3318-3322, Octoher 1987.

[9] W.R. Bennett, C.D. England, D.C. Person, and C.M. Falco, "Magnetic properties of Pd/Co multilayers," J. Appl. Phys., vol. 69, pp. 4384-4390, April 1991.

I10] V. Kaınberský, P. de Hann, J.C. Lodder, J. Simkova, and R. Gemperle, "Dotnain structure in Co-Pd Multilayera," Internag 1993 (to be published).

[11] P. de Haan, Q. Meng, T. Katayama, and J.C. Lodder, "Magnetic and magneto-optical properties of sputtered Co/Pd multilayens," J. Mag. Mag. Mat, vol. 113, pp. 29-35, 1992.

[12] J. Simsová, R. Gemperle, and J.C. Lodder, "The use of colloid -SEM method for domain observation in CoCr films," J. Mag. Mag. Mat., vol. 95, pp. 85-94, 1991.

[13] J. Sim̧̌ova, V. Kamberský, R. Gemperle, J.C. Lodder, W.J.M.A. Geerts, B. Otter, and P. ten Berge, "Domain structure of Co-Cr films on minor loops," J. Mag. Mag. Mat., vol. 101, pp. 196-198, 1991.

[14] W. Andra, "Distrihution of magnetization in multilnyered Films," IEEE Trans. Mag. vol. 2, pp. 560-562, 1966. 